\title{
Feasibility of the TOETVA as an Ambulatory Procedure
}

\author{
Gustavo G Fernández-Ranvier, MD, PhD*, Daniela Guevara, MD, Ghayth Alawwa, MD, \\ Aryan Meknat, MD, Benjamin Lieberman, DO and William B. Inabnet III, MD
}

Department of Surgery, Mount Sinai Hospital, Icahn School of Medicine at Mount Sinai, USA

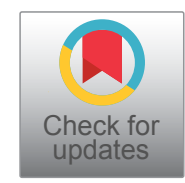

\begin{abstract}
Background: Standard open thyroidectomy has been shown to be feasible and safe as an ambulatory procedure in carefully selected patients. Our aim is to present a protocol with our experience for performing transoral endoscopic thyroidectomy vestibular approach (TOETVA) as an ambulatory procedure that is safe, feasible and applicable as has been shown with open thyroidectomy.

Methods: One hundred and forty patients who underwent a planned ambulatory open thyroidectomy or TOETVA between 2014 and 2019 were identified and retrospectively studied. All patients underwent a similar standardized protocol for same day discharge at the same institution. Outcomes between the two groups including length of stay, operative time, estimated blood loss (EBL), and post-operative complications were compared. Patients were followed from two weeks up to two years post-operatively.

Results: One hundred and forty patients whose age ranges between 18 and 83 were stratified into an open thyroidectomy group and a TOETVA group with 70 patients in each group. In the TOETVA group, 49 (70\%) patients underwent a lobectomy while 21 underwent a total thyroidectomy. One TOETVA was converted to open for RLN injury. Fifty-nine (84\%) patients in the TOETVA group were discharged home on the same day with no complications. There was no significant difference in the average length of stay in the TOETVA group which was $0.2(0-3)$ days compared to $0.3(0-6)$ days in the open group ( $p>0.05$ ). Patients required admission for various reasons: Lack of a caretaker in the immediate post-operative period, lack of access to specialized care, and pain management. There was no significant difference in the EBL, recurrent laryngeal nerve injury, or hypocalcemia post-operatively between the two groups $(p>0.05)$.
\end{abstract}

Conclusion: This investigation shows that TOETVA can be safely performed as an ambulatory procedure in carefully selected patients. It provides equivalent outcomes as the traditional open approach, with no increased risk of postoperative complications and superior cosmetic outcomes.

\section{Keywords}

Thyroid, Thyroidectomy, TOETVA, Ambulatory, Outcomes, Outpatient

\section{Introduction}

The conventional open thyroidectomy via the transcervical route remains the standard of care worldwide for various thyroid diseases [1]. Open thyroidectomy has also been widely accepted as an outpatient procedure, as was emphasized by the American Thyroid Association consensus statement on outpatient thyroidectomy when it is performed on carefully selected patients [1]. Due to improvements in operative technique, the advancement of surgical technology, and outpatient postoperative calcium management, the incidence of dreaded complications such as hematoma formation, bilateral laryngeal nerve palsy, and hypocalcemia have been markedly reduced [2]. The ability to perform the procedure on an outpatient basis decreased cost, nosocomial infection rates, and iatrogenic complications [2].

The transoral endoscopic thyroidectomy vestibular approach (TOETVA) is one of the alternative approaches to per- forming a thyroidectomy without a cutaneous scar through three incisions on the inside of the lower lip that is safe and feasible with ideal cosmetic results [3].

The length of stay after thyroid surgery (open or transoral) is variable depending on the medical condition of the patient, perioperative, geographic, and socio-cultural factors. Length of stay in the United States will vary when compared with

*Corresponding author: Gustavo Fernández-Ranvier, MD, PhD, Division of Metabolic, Endocrine and Minimally Invasive Surgery, Department of Surgery, Mount Sinai Hospital, Icahn School of Medicine at Mount Sinai, USA

Accepted: November 11, 2020

Published online: November 13, 2020

Citation: Fernández-Ranvier GG, Guevara D, Alawwa G, et al. (2020) Feasibility of the TOETVA as an Ambulatory Procedure. J Surgical Endocrinol 2(1):49-54 
other parts of the world [3-5]. As with open thyroidectomy, a customized protocol should be designed to help decide and select patients who may be good candidates for ambulatory TOETVA. The purpose of this study is to present our experience with the TOETVA using our ambulatory surgery protocol and compare outcomes and feasibility with patients undergoing traditional open thyroidectomy.

\section{Materials and Methods}

The research was conducted after approval from the Institutional Review Board. Written consent was obtained by all patients prior to being enrolled in the study. We retrospectively reviewed 140 patients who underwent TOETVA or an open thyroidectomy. Data was reported as mean \pm standard deviation (range). We assessed for significant differences in operative variables and outcomes between those who underwent TOETVA versus an open procedure. Our goal was to identify any statistical differences between the length of stay between TOETVA and open thyroidectomy and to assess safety of the applied ambulatory thyroidectomy protocols. Statistical significance was measured using paired t-tests to generate a two-tailed $p$-value as well as Chi-square values. Significance was defined as a p-value of less than 0.05. Calculations were performed with LaTeX.

Open thyroidectomy was performed with the standard

Table 1: Patient Eligibility for TOETVA.

\begin{tabular}{|l|l|}
\hline Favorable Features & -Patient's own motivation to avoid a cervical scar \\
& -Symptomatic benign nodules $<6 \mathrm{~cm}$ \\
& -Cytologically indeterminate nodules \\
& -Estimate thyroid diameter $<10 \mathrm{~cm}$ on ultrasound \\
& -Estimated gland volume $<45 \mathrm{ml}$ on ultrasound \\
& -Symptomatic Hashimoto's thyroiditis \\
& -Grave's disease \\
& -Differentiated thyroid cancer $<3 \mathrm{~cm}$ without extrathyroidal extension or lymph node metastasis on \\
& preoperative ultrasonography \\
\hline Non-favorable Features & -Substernal goiters \\
& -Previous neck and chin surgery \\
& -Previous neck radiation \\
\hline
\end{tabular}

\begin{tabular}{|c|c|}
\hline $\begin{array}{r}\text { General and } \\
\text { Preoperative } \\
\text { Considerations }\end{array}$ & $\begin{array}{l}\text { - No significant medical comorbidities such as } \\
\text {-Uncompensated cardiac or respiratory disease } \\
\text {-ESRD } \\
\text {-Anticoagulation or Antiplatelets } \\
\text { - Visual or Hearing Impairments } \\
\text { - OSA } \\
\text { - Pregnancy } \\
\text { - Caregiver is accessable and capable or understanding post-op } \\
\text { instructions } \\
\text {-Patient and caregiver preoperative education and } \\
\text { understanding }\end{array}$ \\
\hline $\begin{array}{l}\text { Intraoperative } \\
\text { Considerations }\end{array}$ & $\begin{array}{l}\text { - Lack of the following pathologic characteristics } \\
\text {-Massive goiters } \\
\text {-Extensive retrosternal extension } \\
\text { - Locally advanced malignancy } \\
\text {-Parathyroid gland identified and preserved } \\
\text { - Nerve monitoring confirms integrity of RLN } \\
\text {-Safe surgical technique with careful hemostasis }\end{array}$ \\
\hline $\begin{array}{l}\text { Postoperative } \\
\text { considerations }\end{array}$ & $\begin{array}{l}\text { - Clinical vocal cord function assessment } \\
\text { - Oral outpatient calcium for total thyroidectomy and } \\
\text { completion thyroidectomy } \\
\text {-East and fast access to the hospital } \\
\text { - Direct communication with the surgeon } \\
\text { - Caregiver company for the first night }\end{array}$ \\
\hline
\end{tabular}

Figure 1: Ambulatory TOETVA protocol. 
Citation: Fernández-Ranvier GG, Guevara D, Alawwa G, et al. (2020) Feasibility of the TOETVA as an Ambulatory Procedure. J Surgical Endocrinol 2(1):49-54

transcervical approach whereas TOETVA was completed using the technique as previously described [6]. One surgeon performed only open thyroidectomies, two surgeons performed only TOETVA, and one surgeon performed both.

Firstly, after determining who could be a good candidate for TOETVA as indicated in Table 1 [6], the process of selecting patients that would be good candidates for ambulatory TOETVA needs to be further evaluated. This is based on multiple factors that include preoperative considerations that focus on the patients' medical condition and social factors, intraoperative considerations regarding the characteristics of the thyroid gland or nodule and the operative course, as well as postoperative factors to assess for development of major complications. All these considerations play a role in a successful outpatient thyroidectomy and are summarized in Figure 1 [1].

After successfully fulfilling all the above criteria, if the operative course was uncomplicated, patients are observed for 3 hours as follows:

- 2 hours in the immediate recovery area with cardiorespiratory monitoring

- 1 hour in a "step-down" recovery area where the patient is placed on a per oral (P.O.) diet and ambulates

- Patient is discharged home after evaluation by the attend- ing surgeon following a pre-established checklist:

1. Normal vital signs

2. Normal voice

3. No respiratory distress or abnormalities

4. No neck swelling

5. Nausea and pain are controlled

6. Patient can ambulate

7. P.O. diet has been tolerated

\section{Results}

Data of 140 patients who underwent thyroidectomy via transcervical or TOETVA from a single institution were included in this investigation. The data was stratified into two groups based on the approach of the procedure performed into an open thyroidectomy $(n=70)$ and a TOETVA $(n=70)$ group.

Details about patients' population and demographics is described in Table 2 . The mean age of the TOETVA and open thyroidectomy groups was 47 and $50 \pm 2.1(18-83)$, respectively. Sixty-three patients (90\%) and fifty-five patients (79\%) of the TOETVA and open thyroidectomy groups were female, respectively. Time to follow up varied from several weeks postoperatively up to two years.

Table 2: Demographic data.

\begin{tabular}{|c|c|c|c|}
\hline & TOETVA & Open thyroidectomy & Total \\
\hline Variables [mean \pm stdev (range)] & $(n=70)$ & $(n=70)$ & $(n=140)$ \\
\hline Age & $47 \pm 13.5(31-70)$ & $50 \pm 17.4(18-83)$ & $48.5 \pm 2.1(18-83)$ \\
\hline Male & $48 \pm 13.6(32-69)$ & $48.1 \pm 15.6(22-74)$ & $48.05 \pm 0.07(22-74)$ \\
\hline Female & $46 \pm 13.4(24-70)$ & $50.9 \pm 17.9(16-83)$ & $48.45 \pm 3.5(16-83)$ \\
\hline \multicolumn{4}{|l|}{ Gender } \\
\hline Male & $7(10 \%)$ & $15(21.4 \%)$ & $22(15.7 \%)$ \\
\hline Female & $63(90 \%)$ & 55 (78.6\%) & $118(84.3 \%)$ \\
\hline BMI $\left(\mathrm{kg} / \mathrm{m}^{2}\right)$ [mean \pm stdev] & $26 \pm 5.6$ & $28.7 \pm 6.8$ & $27.35(19.5 \%)$ \\
\hline \multicolumn{4}{|l|}{ Pre-operative diagnosis } \\
\hline \multicolumn{4}{|l|}{ Nodules/Multinodular Goiters } \\
\hline BI & 0 & 0 & 0 \\
\hline B II & $34(49.0 \%)$ & $46(65.7 \%)$ & 79 (56.4\%) \\
\hline B III & $14(20 \%)$ & $3(4.3 \%)$ & $17(12.1 \%)$ \\
\hline B IV & $6(8.6 \%)$ & 0 & $6(4.3 \%)$ \\
\hline B V & $4(5.7 \%)$ & 0 & $4(2.8 \%)$ \\
\hline B VI & 12 (14.1\%) & $12(17.1 \%)$ & $24(17.1 \%)$ \\
\hline \multicolumn{4}{|l|}{ Symptomatic benign disease } \\
\hline Grave's disease & $8(11.4 \%)$ & $1(1.4 \%)$ & $2(1.4 \%)$ \\
\hline Hashimoto's thyroiditis & $1(1.4 \%)$ & $7(10 \%)$ & $15(10.7 \%)$ \\
\hline Tumor size (mm) [mean (range)] & $2.3(7-60)$ & $2.2(0.01-5.6)$ & $2.25(1.6 \%)$ \\
\hline
\end{tabular}

BMI: Body Mass Index; B: Bethesda Classification; stdev: Standard Deviation. 
The indication for surgery was variable and included thyroid nodules, multinodular goiters, Graves' disease, and papillary thyroid carcinoma. Preoperatively nodule size was similar in both groups with a mean size of $2.3(0.7-6) \mathrm{cm}$ in the TOETVA and $2.2(0.1-5.6) \mathrm{cm}$ in the TOETVA and open thyroidectomy groups respectively. Forty-nine (70\%) patients underwent a transoral lobectomy, and forty-three (61.4\%) patients underwent an open lobectomy, with the remaining undergoing total thyroidectomy.

There was no significant difference in the length of stay, with most patients going home within 24 hours of the procedure, $0.2(0-3)$ days in the TOETVA group vs. $0.3(0-6)$ days in the open group, $p>0.05$ ). Ten patients in the TOETVA group who did not meet the criteria postoperatively for a safe ambulatory discharge (14\%) required an overnight stay, and one patient $(1 \%)$ required admission for three nights for pain control. Nine patients in the Open group did not meet criteria for safe ambulatory discharge, and one patient required admission for six days due to treatment of hypocalcemia.

The operative time in minutes for TOETVA was compared to open thyroidectomy when performing a lobectomy (151 \pm $36.3(90-256)$ vs. $85.8 \pm 26(51-150))$ and total thyroidectomy $(231.3 \pm 40.8$ (175-344) vs. $112.1 \pm 33.8$ (64-195)), $\mathrm{p}<0.05$. The estimated blood loss for the TOETVA group was $27.5 \pm$ $62.3 \mathrm{ml}$ compared to $29.7 \pm 3.9 \mathrm{ml} \mathrm{p}=\mathrm{NS}$ ). Operative details can be found in Table 3.

There was once case of TOETVA that required conversion to an open procedure due to transection of the recurrent laryngeal nerve and subsequent repair. One patient had a small tracheal perforation secondary to Veress needle insertion, which was also repaired intraoperatively and the patient was discharged safely on the same day without conversion. Two patients in the TOETVA group (2.8\%) had a recurrent laryngeal nerve injury compared to none in the open group. Both patients were discharged on the same day and followed up closely with good resolution of symptoms. Eighteen patients (25.7\%) of the TOETVA group complained of lower lip numbness postoperatively, which required a mean of 24 weeks to recover. Chin numbness was also seen in 15 patients $(21.4 \%)$ of the TOETVA group postoperatively, which required a mean of 16 weeks to complete recovery. All lower lip and chin numbness recovered and did not significantly impact patient's quality of life. There was no significant difference between the rates of temporary hypoparathyroidism, which was encountered in two patients $(2.8 \%)$ in the TOETVA group and one patient (1.4\%) in the open group. None of the patients in either group developed permanent hypoparathyroidism.

\section{Discussion}

In recent years there has been increasing emphasis on the benefits of performing outpatient surgery. Performing surgery without requiring inpatient postoperative management decreases the rates of nosocomial infection, iatrogenic complications, and cost, improves patient comfort, and conserves resources [2]. The same trends apply to thyroid surgery. When performing any outpatient procedure, the main concern remains patient safety and formulating criteria for a safe discharge.

Outpatient thyroid surgery has been performed and studied thoroughly over the last decade with favorable outcomes. There are clearly defined parameters regarding patient selection, preoperative, intraoperative, and postoperative factors that play a role in a successful outpatient thyroidectomy $[2,7]$. The same rules and parameters can be applied to the TOETVA approach.

Two series of patients who underwent transoral thyroidectomy, one in the United States and one in Italy, were published where patients were discharged after being observed overnight without adverse complications [4,5]. Asian countries document a longer length of a stay where it is more com-

Table 3: Operative details.

\begin{tabular}{|c|c|c|c|c|}
\hline & TOETVA & Open thyroidectomy & Total & $P$ value \\
\hline & $(n=70)$ & $(n=70)$ & $(n=140)$ & \\
\hline \multicolumn{5}{|l|}{ Number of Cases } \\
\hline Lobectomy & 49 (70\%) & $24(34.3 \%)$ & $73(52.1 \%)$ & \\
\hline Total & $21(30 \%)$ & $43(61.4 \%)$ & $64(45.7 \%)$ & \\
\hline Completion & 0 & $3(4.3 \%)$ & $3(2.1 \%)$ & \\
\hline \multicolumn{5}{|c|}{ Operative time (mins) [mean \pm stdev (range)] } \\
\hline Lobectomy & $151 \pm 36.3(90-256)$ & $85.8 \pm 26(51-150)$ & $118.4 \pm 46.1(51-256)$ & $<0.05$ \\
\hline Total & $231.3 \pm 40.8(175-344)$ & $112.1 \pm 33.8(64-195)$ & $171.7 \pm 84.3(64-344)$ & $<0.05$ \\
\hline Completion & 0 & $133 \pm 13.7(121-148)$ & $133 \pm 13.7(121-148)$ & \\
\hline \multicolumn{5}{|l|}{ Final pathology } \\
\hline Benign & $36(51.4 \%)$ & $43(61.4 \%)$ & 79 (56.4\%) & \\
\hline PTC & $34(48.6 \%)$ & $27(38.6 \%)$ & $61(43.6 \%)$ & \\
\hline EBL $(m L)$ [mean \pm range] & $27.5 \pm 62.3$ & $29.7 \pm 3.9$ & $28.6 \pm 1.5$ & 0.4 \\
\hline LOS (days) [mean (range)] & $0.2(0-3)$ & $0.3(0-6)$ & $0.25(0-6)$ & 0.5 \\
\hline
\end{tabular}

PTC: Papillary Thyroid Carcinoma; EBL: Estimated Blood Loss; LOS: Length of Stay; stdev: Standard Deviation 
Table 4: Complications.

\begin{tabular}{|c|c|c|c|c|}
\hline & TOETVA $(n=70)$ & $\begin{array}{l}\text { OPEN THYROIDECTOMY } \\
\qquad(n=70)\end{array}$ & Total $(n=140)$ & $P$ value \\
\hline Tracheal perforation & $1(1.4 \%)$ & 0 & $1(0.7 \%)$ & 0.09 \\
\hline Lower lip Numbness & $18(25.7 \%)$ & 0 & $18(12.8 \%)$ & \\
\hline Mean time to recovery (weeks) & $23.8(0.43-48)$ & & $23.8(17 \%)$ & \\
\hline Persistent numbness & $1(1.4 \%)$ & & $1(0.7 \%)$ & \\
\hline Chin numbness & $15(21.4 \%)$ & 0 & $15(10.7 \%)$ & \\
\hline Mean time to recovery (weeks) & $15.7(2-48)$ & & $15.7(11.2 \%)$ & \\
\hline Persistent numbness & 0 & & & \\
\hline Temporary hypoparathyroidism & $2(2.8 \%)$ & $1(1.42 \%)$ & $3(2.1 \%)$ & 0.21 \\
\hline Persistent hypoparathyroidism & 0 & 0 & 0 & \\
\hline Temporary RLN injury & $2(2.8 \%)$ & 0 & $2(1.4 \%)$ & 0.18 \\
\hline Permanent RLN injury & 0 & 0 & 0 & \\
\hline Post-operative infections & $2(2.8 \%)$ & 0 & $2(1.4 \%)$ & 0.18 \\
\hline Mean time to follow up (months) & $3.2(0.25-14)$ & $9.4(0.7-54)$ & $6.3(0.25-54)$ & \\
\hline
\end{tabular}

mon for the patients to have a longer recovery period as an inpatient rather than transitioning directly to homecare even when they are medically cleared for discharge [8].

The main concerns to adopting an ambulatory thyroidectomy approach are the potential to develop postoperative hemorrhage, bilateral laryngeal nerve paralysis, and/or severe hypocalcemia [2]. All three represent potential life - threatening complications and if developed, they may necessitate monitoring in an inpatient setting. Improved hemostatic techniques, improved recurrent laryngeal nerve monitoring, and calcium supplementation postoperatively have been cited to be major contributors to making this procedure feasible as an outpatient surgery [9]. In our experience, this protocol has been proven to be safe in our patients who underwent open thyroidectomy. The protocol begins with careful patient selection. It is critical that the patient and the surgeon develop a strong partnership with clear discussions regarding what the patient should expect on the day of the procedure and during the postoperative course. The patient should also be provided with educational resources to help enrich the educational process even after the encounter has ended $[2,7]$.

Our study cohort contained 70 patients in the open thyroidectomy group and 70 patients in the TOETVA group. All patients were carefully selected by the attending surgeon to be candidates for same-day discharge. Eighty-five percent of patients from both groups were discharged on the same day of surgery. None of these patients in the open or TOETVA groups developed life-threatening complications. Patients that required an overnight stay after TOETVA or open thyroidectomy did not fit the discharge criteria for various reasons. The patients lived in an area where they did not have fast access to a facility capable of managing postoperative complications, did not have sufficient social support, had oral discomfort, were unable to tolerate an oral diet, or their procedure finished at a late time in the evening when we considered not a safe time for discharge.

The duration of surgery was significantly longer in the
TOETVA group compared to the open group, which is accounted for by the extra time required for port placement and the creation of the workspace. It is also a relatively new procedure with a steep learning curve compared to the open approach that has been performed for many decades.

The acceptable prevalence of postoperative hemorrhage after a standard open thyroidectomy is approximately $1 \%$ [9]. None of the patients in the open or TOETVA groups suffered from significant bleeding.

Injury to the recurrent laryngeal nerve is a well-established complication after thyroidectomy. The clinical significance of such an injury is broad. It can range from undetectable hoarseness that is seen after minor unilateral recurrent nerve injuries to airway compromise due to bilateral vocal fold paralysis and airway obstruction that may require reintubation or emergent tracheostomy. The overall risk of permanent symptomatic recurrent nerve injury varies from $0.8 \%$ to $3 \%$. In the TOETVA group two patients suffered from recurrent laryngeal nerve injuries that manifested as hoarseness at 3 and 5 months follow up compared to none in the open group.

Hypocalcemia is also another cause of prolonged hospitalization after thyroid surgery. Transient hypocalcemia may occur in $25 \%$ of patients undergoing bilateral thyroid surgery and is permanent in $0.9-14 \%$ of cases. We routinely supplemented calcium and vitamin D postoperatively. None of our patients in both the TOETVA and open thyroidectomy groups developed clinically significant hypoparathyroidism.

Apart from the standard complications faced postoperatively after thyroidectomy, TOETVA also presents unique complications that are not seen with the open approach including mental nerve injuries resulting in numbness below the lip, carbon dioxide embolism, full-thickness skin injury, and cutaneous lesions of the chin and neck that need to be considered [10]. Eighteen patients (25.7\%) of the TOETVA group complained of lower lip numbness postoperatively, which re- 
quired a mean of 24 weeks to recover. Chin numbness was also seen in 15 patients (21.4\%) of the TOETVA group postoperatively, which required a mean of 16 weeks to complete recovery. These complications, however, did not hinder safe same day discharge.

\section{Conclusion}

This investigation suggests that TOETVA can be safely performed as an ambulatory procedure. Patient selection is an integral part of the process. TOETVA has become a feasible and safe option that provides equivalent outcomes to the standard open thyroidectomy with no increased risk of postoperative complications and superior cosmetic outcomes.

\section{Disclosures}

This project was supported in part by grant $T L-$ 1TR0014341 from the National Center for Advancing Translational Sciences, National Institutes of Health.

\section{References}

1. Terris DJ, Samuel Snyder, Denise Carneiro-Pla, et al. (2013) American thyroid association statement on outpatient thyroidectomy. Thyroid 23: 1193-202.

2. Segel JM, Duke WS, White JR, et al. (2016) Outpatient thyroid surgery: Safety of an optimized protocol in more than 1,000 patients. Surgery 159: 518-23.
3. Anuwong A, Ketwong K, Jitpratoom P, et al. (2018) Safety and outcomes of the transoral endoscopic thyroidectomy vestibular approach. JAMA Surg 153: 21-27.

4. Dionigi G, Bacuzzi A, Lavazza M, et al. (2017) Transoral endoscopic thyroidectomy: Preliminary experience in Italy. Updates Surg 69: 225-234.

5. Witzel K, Messenbaeck F, Weitzendorfer M, et al. (2017) Transoral thyroidectomy: Limitations, patients' safety, and own experiences. Updates Surg 69: 193-198.

6. Fernandez-Ranvier G, Meknat A, Guevara DE, et al. (2019) Transoral endoscopic thyroidectomy vestibular approach. JSLS 23.

7. Russell JO, Christopher R Razavi, Mohammad Shaear, et al. (2019) Transoral vestibular thyroidectomy: Current state of affairs and considerations for the future. J Clin Endocrinol Metab 104: 3779-3784.

8. Anuwong A, Spagnol M, Sanabria A, et al. (2018) Transoral endoscopic thyroidectomy vestibular approach (TOETVA): Indications, techniques and results. Surg Endosc 32(1): 456-465.

9. Lewis Flint RP (2015) Endocrine surgery. Selected Readings in General Surgery 41.

10. Camenzuli C, P Schembri Wismayer, J Calleja Agius (2018) Transoral endoscopic thyroidectomy: A systematic review of the practice so far. JSLS 22. 